\title{
A Vital Wearing System with Wireless Capability
}

\author{
Mu-Huo Cheng, Li-Chung Chen, Ying-Che Hung, Chien-Nan Chen \\ Dept. of Electrical and Control Engineering \\ National Chiao Tung University \\ 1001, Ta Hsieh Rd., Hsinchu, Taiwan 300 \\ Email: mhcheng@cc.nctu.edu.tw
}

\author{
Chang Ming Yang, Tzu Lin Yang \\ Ming Young Biomedical Corp. \\ No. 27, Guangfu Rd., Jhunan \\ Miaoli 350,Taiwan \\ WWW: http://www.my-cares.com \\ Email:info@my-cares.com
}

\begin{abstract}
A system with wearable sensors for detecting and sensing vital and physical motion signals such as the ECG, respiration, temperature, sweatiness, three-axis accelerations, and the motions of both knee ankles and elbows is presented. The system is also equipped with a bluetooth transceiver such that the obtained sensor signals can be transmitted to mobile phones or remote computers for either monitoring or post signal processing to extract knowledges or information. The presented system is embedded with signal processing algorithms such as the heart rate, the respiratory period, the body temperature, the degree of sweatiness, and the posture of wearing person are demonstrated. The heart rate and heart rate variation from the measured ECG data are estimated using an adaptive ESPRIT algorithm. This system is highly suitable for applications of remote healthcare and wellness.
\end{abstract}

\section{INTRODUCTION}

Since the world population is getting older, the need for a comfortably wearable system with capability to measure and wireless transmit vital signals is becoming more urgent [1]. The wearable sensors especially made of textiles with suitable materials have been flourishing recently. For example, using the wearable medical clothes for monitoring respiration activity and ECG signal [2], for tele-home healthcare [3], for neurological rehabilitation [4] have been proposed recently. Extensive research effort for developing wearable medical devices have been started such as the VTAMN project in France, the WEALTHY project in Europe, and the LifeShirt in USA [5]. In this work, we present a vital wearing system with clothes sewn with sensors capable of measuring ECG signals, the respiration activity, the body temperature, the degree of sweatiness, and the motions of important joints such as knee ankles and elbows. The steel textile is used to realize the ECG electrodes and the fabric-based strain-gauge is developed to implement the respiration and motion sensors. In addition to the clothes, the system only needs a small control box containing

Permission to make digital or hard copies of all or part of this work for personal or classroom use is granted without fee provided that copies are not made or distributed for profit or commercial advantage and that copies bear this notice and the full citation on the first page. To copy otherwise, to republish, to post on servers or to redistribute to lists, requires prior specific permission and/or a fee PERVASIVEHEALTH 2008, 30 Jan - 1 Feb. Tampere, Finland

Copyright $\odot 2008$ ICST 978-963-9799-15-8

DOI 10.4108/ICST.PERVASIVEHEALTH2008.2947 conditioning circuits, a bluetooth transceiver, and threeaxis accelerators. The clothes is soft and comfortable due to the usage of textile sensors and it is durable. The received data can be further processed to extract more meaningful information. In this paper, we apply the well-known adaptive estimation of signal parameters via rotational invariance techniques (ESPRIT) [7] to estimate the heart rate adaptively. Simulations demonstrate that this algorithm can successfully estimate the heart rate and its variability from the received ECG data when the wearing person begins with sitting still to walking through jogging.

\section{ThE System}

The presented system is divided into two parts: one is the textile clothing knitted with various sensors and the other is a small control box used for signal integration, receiving, and transmission. One shirt sewn with vital sensors and the control box are shown in Fig. 1. The shirt shown contains ECG sensor electrodes, a respiratory fabric-based belt sensor, a temperature sensor, and a sweatiness sensor, all sewn in the clothes such that the wearing person can feel comfortable. The control box includes signal conditioning circuits, a three-axis accelerometer and a bluetooth transceiver; the signal conditioning circuits contains some anti-aliasing filters, noise filters and comparators, the three-axis accelerometer is used to measure the motions of the wearing person, and the bluetooth transceiver is to receive remote signals as well as to transmit the data obtained from various sensors to remote mobile phones or computers for further applications.

The receiver LabVIEW interface of the remote computer via bluetooth wireless transmission for typical measured data is shown in Fig. 2 where the two plots on the left depict the ECG and respiratory signals, the three indicators on the lower left show the degree of sweatiness, the body temperature, and the room temperature, 


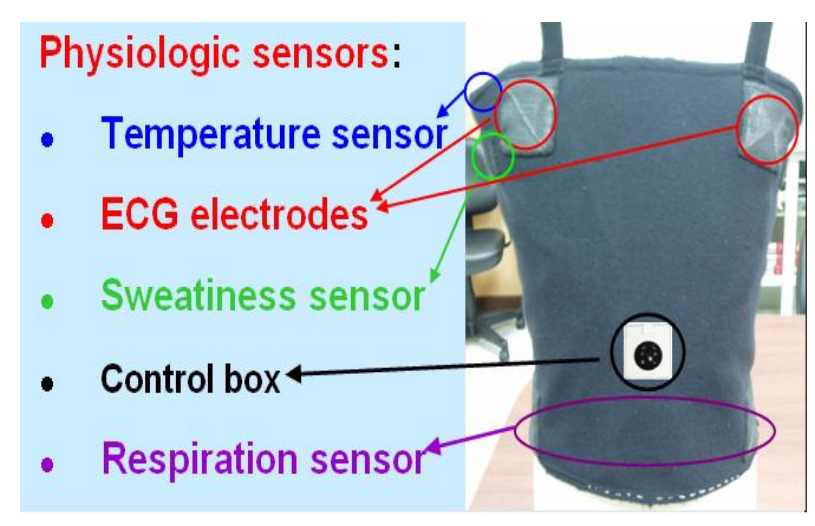

Fig. 1. The presented vital wearing system

the three meters on the upper right show the three-axis accelerations of the wearing person, and the pictured figure on the lower right illustrates the posture of the wearing person.

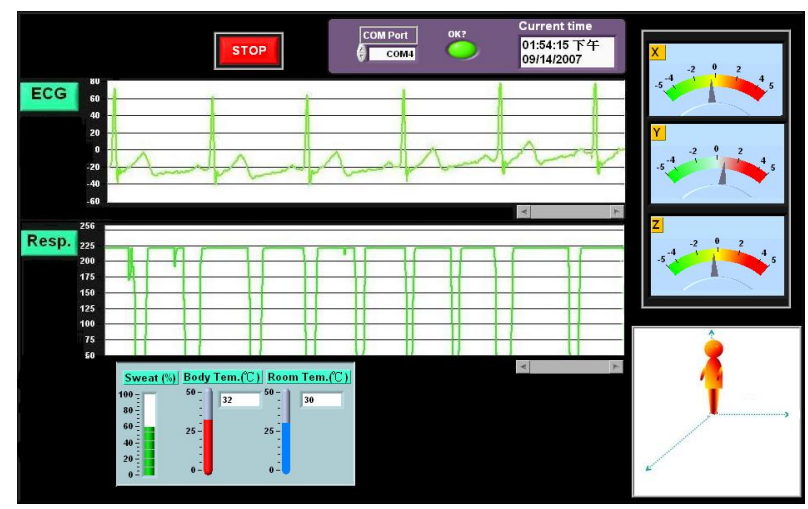

Fig. 2. The LabVIEW interface of the remote computer for typical measured data

The equipped sensors and its measured data received by a computer via bluetooth wireless transmission are discussed in detail below.

\section{A. ECG Sensor}

The ECG sensor in this system has two electrodes realized by the steel textile. The signals from the electrodes are passed through filters with their specifications conformed to the American National Standard [6]. The filtered signals are sampled via an analog/digital converter at the rate of 200 samples per second and then transmitted by bluetooth to the remote computer. We present three measured ECG signals with the wearing person sitting still, walking steadily, or jogging, showing that effective ECG data can be obtained even the sensor is sensitive to motions of the wearing person. Fig. 3 shows the obtained ECG signals; the upper signals are obtained with the wearing person sitting still, the middle signals with the person steadily walking, and the lower one with the person jogging. These signals clearly demonstrate that the measured ECG signals contain few interferences for the first two cases; when the wearing person is jogging, however, the measured ECG signals demonstrate severe interferences. Even when the wearing person is jogging, we observe that the $\mathrm{R}$ peaks in the measured ECG are always present. Hence, we can correctly estimate the heart rate from the presence of $\mathrm{R}$ peaks in ECG signals. Moreover, the signal processing technique, discussed later, can estimate the heart rate adaptively such that the heart-rate variability can be obtained.

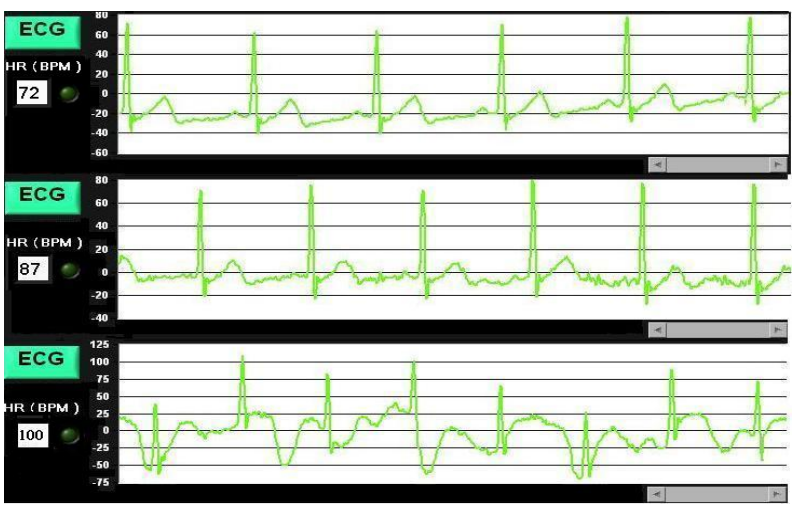

Fig. 3. The received ECG data with wearing person sitting still, walking, and jogging

\section{B. Respiration Sensor}

The respiration sensor in this system is realized by a belt made of fabric-based strain-gauge; the belt resistance varies due to the elongation caused by the respiratory motion. The belt can be easily sewn into the clothes. Present system only detects the on-off motion by comparing the belt resistance with a threshold value such that the respiration corresponds to an on-off motion.

\section{Three-Axis Accelerators, Temperature, Sweatiness and Motions}

The three-axis accelerometer integrated circuit is mounted on the control box which normally is carried along the waist. The body temperature sensor is obtained via a thermistor knitted in the clothes and connected using the steel fiber to the control box. The sweatiness sensor is realized by the resistance of steel textile varying with the degree of sweat. Finally, the idea of realizing the respiratory sensor is used to realize the motion sensor; 
the fabric-based strain-gauge, properly located near the joint position, can correspond its resistance effectively to the degree of motion.

\section{Post Signal Processing}

The purpose of post signal processing is to use the measured data for extracting meaningful information or knowledges which can either help doctor diagnose or judge the physical status of the wearing person whether calling emergency help is needed. This system has been equipped with signal processing capabilities to obtain the heart rate from the ECG. The respiration period can be similarly obtained. The posture of the wearing person derived from the three axes accelerators and the motion detectors of important joints such as knee ankles, shoulder, waist, and elbows are still developing. The adaptive algorithm for computing the heart rate and its variability from ECG data is discussed below.

\section{A. Adaptive ESPRIT}

ESPRIT algorithm is known an effective algorithm for estimating signal frequencies using the subspace information. The adaptive ESPRIT development lies on the design of subspace tracker. In this work we use the orthonormal PAST (OPAST) subspace tracking algorithm [8] to develop the adaptive ESPRIT for estimating the heart rate from the measured data.

Due to space limit, we briefly introduce the developed algorithm. The OPAST subspace tracking algorithm will output a subspace matrix $W_{o}(t)$ using the measured ECG data at the sample time $t$ by an update equation given by

$$
W_{o}(t)=W_{o}(t-1)+\boldsymbol{p}(t) \boldsymbol{q}^{H}(t)
$$

Denote

$$
\begin{gathered}
W_{o}(t)=\left[\begin{array}{l}
V_{x}(t) \\
V_{y}(t)
\end{array}\right] \\
\boldsymbol{p}(t)=\left[\begin{array}{l}
\boldsymbol{p}_{x}(t) \\
\boldsymbol{p}_{y}(t)
\end{array}\right]
\end{gathered}
$$

Define two matrix products $H_{1}(t) \in \mathcal{C}^{r \times r}$ and $H_{2}(t) \in$ $\mathcal{C}^{r \times r}$ below

$$
\begin{aligned}
& H_{1}(t)=V_{x}^{H}(t) V_{x}(t) \\
& H_{2}(t)=V_{x}^{H}(t) V_{y}(t)
\end{aligned}
$$

The ESPRIT algorithm turns out to find the eigenvalues of the matrix $\Psi(t)=\left[H_{1}(t)\right]^{-1} H_{2}(t)$ where $H_{1}(t)$ and
TABLE I

\section{ADAPTIVE ESPRIT ALGORITHM}

\begin{tabular}{l}
\hline Equation \\
\hline Initialize: \\
$H_{1}(0)=V_{x}^{H}(0) V_{x}(0)$ \\
$H_{2}(0)=V_{x}^{H}(0) V_{y}(0)$ \\
\hline Output from Subspace Tracker: \\
$W_{o}(t-1), \boldsymbol{p}(t), Q_{r}(t)$ for $t=1,2, \cdots$ \\
\hline Partitions: \\
$W_{o}(t-1)=\left[\begin{array}{c}V_{x}(t-1) \\
V_{y}(t-1)\end{array}\right]$ \\
$\boldsymbol{p}(t)=\left[\begin{array}{c}\boldsymbol{p}_{x}(t) \\
\boldsymbol{p}_{y}(t)\end{array}\right]$ \\
\hline$p_{1}(t)=\left[\boldsymbol{p}_{x}(t)\right]^{H} \boldsymbol{p}_{x}(t)$ \\
$p_{2}(t)=\left[\boldsymbol{p}_{x}(t)\right]^{H} \boldsymbol{p}_{y}(t)$ \\
$H_{1}(t)=H_{1}(t-1)+V_{x}^{H}(t-1) \boldsymbol{p}_{x}(t) \boldsymbol{q}^{H}(t)$ \\
$+\boldsymbol{q}(t)\left[\boldsymbol{p}_{x}(t)\right]^{H} V_{x}(t-1)+p_{1}(t) Q_{r}(t)$ \\
$H_{2}(t)=H_{2}(t-1)+V_{x}^{H}(t-1) \boldsymbol{p}_{y}(t) \boldsymbol{q}^{H}(t)$ \\
$+\boldsymbol{q}(t)\left[\boldsymbol{p}_{x}(t)\right]^{H} V_{y}(t-1)+p_{2}(t) Q_{r}(t)$ \\
$\Psi(t)=\left[H_{1}(t)\right]^{-1} H_{2}(t)$ \\
\hline
\end{tabular}

$H_{2}(t)$ can be updated by direction substitution using (2)(3) into (1), yielding

$$
\begin{aligned}
H_{1}(t) & =\left[V_{x}(t-1)+\boldsymbol{p}_{x}(t) \boldsymbol{q}^{H}(t)\right]^{H}\left[V_{x}(t-1)+\boldsymbol{p}_{x}(t) \boldsymbol{q}^{H}(t)\right] \\
& =H_{1}(t-1)+V_{x}^{H}(t-1) \boldsymbol{p}_{x}(t) \boldsymbol{q}^{H}(t) \\
& +\boldsymbol{q}(t)\left[\boldsymbol{p}_{x}(t)\right]^{H} V_{x}(t-1)+p_{1}(t) Q_{r}(t) \\
H_{2}(t) & =\left[V_{x}(t-1)+\boldsymbol{p}_{x}(t) \boldsymbol{q}^{H}(t)\right]^{H}\left[V_{y}(t-1)+\boldsymbol{p}_{y}(t) \boldsymbol{q}^{H}(t)\right] \\
& =H_{2}(t-1)+V_{x}^{H}(t-1) \boldsymbol{p}_{y}(t) \boldsymbol{q}^{H}(t) \\
& +\boldsymbol{q}(t)\left[\boldsymbol{p}_{x}(t)\right]^{H} V_{y}(t-1)+p_{2}(t) Q_{r}(t)
\end{aligned}
$$

where

$$
\begin{aligned}
p_{1}(t) & =\left[\boldsymbol{p}_{x}(t)\right]^{H} \boldsymbol{p}_{x}(t) \\
Q_{r}(t) & =\boldsymbol{q}(t) \boldsymbol{q}^{H}(t) \\
p_{2}(t) & =\left[\boldsymbol{p}_{x}(t)\right]^{H} \boldsymbol{p}_{y}(t)
\end{aligned}
$$

The summary of our adaptive ESPRIT approach for estimating heart rate from the ECG is given in Table I.

\section{B. One Experiment}

One experiment is presented to show the effectiveness of the adaptive algorithm. The ECG data are measured with the wearing person varying from initially sitting still to walking steadily until jogging. The adaptive ESPRIT is then applied to estimate the heart rate from these data. Fig. 4 depicts two plots; the upper plot shows the measured ECG data of about 10 seconds and the lower plot shows the estimated frequencies from the algorithm. These results indicate that the heart rate is 
not varying when the person is either sitting or walking. When the person begins jogging, however, the heart rate is increased tremendously and the adaptive algorithm successfully obtains estimate of heart rate.
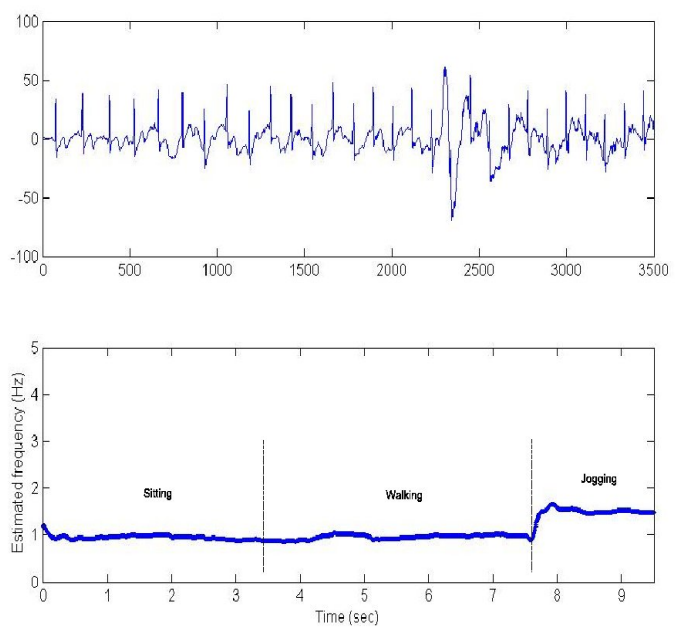

Fig. 4. The received ECG data and the obtained heart rate estimates using adaptive ESPRIT

\section{CONCLUSiOnS}

A vital wearing system is presented in this paper. The clothes is sewn with steel textile for realizing ECG sensors and fabric-based strain gauge for respiration and motion sensors. The sensors to measure the body temperature and degree of sweatiness are also embedded. In addition to the clothes, a small control box with mounted a three-axis accelerometer and a bluetooth transceiver such that the posture of wearing person can be detected and wireless transmission of data is realized. This system can be used for remote health monitoring and diagnosis. This system is especially useful for reducing the incidence of life-style related and chronic diseases; it also can be used for self-care and management of patient's health. We also think this system can make patient's physiologic signals easy to access, increasing the quality of healthcare.

\section{ACKNOWLEDGMENT}

This work was supported by National Science Council, Taiwan, under NSC96-2221-E-009-013 and NSC962622-E-009-012.

\section{REFERENCES}

[1] S. Park and S. Jayaraman, "Enhancing quality of life through wearable technology," IEEE Eng. in Medicine and Biology Magazine, pp. 41-48, May/June 2003.
[2] R. Paradiso, A. Gemingnani, E. P. Scilingo, and E. De Rossi, "Knitted bioclothes for cardiopulmonary monitoring," Proc. of 25th Int. Conf. IEEE EMBS, pp. 3720-3723, 2003.

[3] K. Hung, Y. T. Zhang, and B. Tai, "Wearable medical devices for tele-home healthcare," Proc. of 26th Int. Conf. IEEE EMBS, San Francisco, pp. 5384-5387, 2004.

[4] T. Giorgino, F. Lorussi, D. De Rossi, and S. Quaglini, "Posture classification via wearable strain sensors for neurological rehabilitation," Proc. of 28th Int. Conf. IEEE EMBS, New York, pp. 6273-6276, 2006.

[5] F. Axisa, P. M. Schmitt, C. Gehin, G. Delhomme, E. McAdams, and A. Dittmar, "Flexible technologies and smart clothing for citizen mdeicine, home healthcare, and disease prevention," IEEE Trans. Information Technology in Biomedicine, vol. 9, no. 3, pp. 325-336, Sept. 2005.

[6] American National Standard, Cardiac monitors, heart rate meters, and alarms, ANSI/AAMI EC 13:2002

[7] R. Roy and T. Kailath, "ESPRIT-Estimation of Signal Parameters via Rotational Invariance Techniques," IEEE Trans. Acoust., Speech, Signal Processing, vol. 37, no. 7, pp.984-995, July 1989.

[8] K. Abed-Meraim, A. Chkeif, and Y. Hua, "Fast Orthonormal PAST Algorithm," IEEE Signal Processing Letters, vol. 7, no. 3, pp. 60-62, Mar. 2000 